\title{
A Compact Wide Bandpass Filter for Satellite Communications with Improved Out-of-Band Rejection
}

\author{
Badiaa Ait Ahmed $^{1}$, Azzeddin Naghar ${ }^{2}$, Otman Aghzout ${ }^{1}$, Ana Vazquez Alejos ${ }^{3}$ and Francisco Falcone ${ }^{4}$ \\ ${ }^{1}$ Department of Computer Science Engineering, SIGL Lab. ENSA, \\ University of Abdelmalek Essaadi ,Tetuan, Morocco \\ 2 BSA-Innovation LAB Radio Frequency Systems RFS, Lannion,France \\ ${ }^{3}$ Department of Signal Theory and communications, \\ University of Vigo, Pontevedra, Spain \\ ${ }^{4}$ Department of Electrical and Electronic Engineering, \\ Public University of Navarra,Pamplona, Spain \\ *corresponding author, E-mail: oagh zout @uae.ac.ma
}

\begin{abstract}
This paper presents a compact C-band bandpass filter with improved out-of-band rejection. It consists of a symmetric three-pole parallel coupled line microstrip loaded with complementary split ring resonators $(C S R R s)$ and slot resonators. Enhancements regarding the filter responses are obtained by etching these two parasitic shapes successively. When, $C S R R s$ are introduced in the ground plane, exactly in the back of the transmission line of $50 \Omega$, improvements on the filter selectivity level are achieved. Whereas, the slot resonator elements are implemented on the feed line to permit the suppression of the second harmonics. To reduce the second harmonics of $11.1-13 \mathrm{GHz}$, the parasitic elements are both combined, widen the filter rejection of $5.81 \%$. The surface current distribution together with the equivalent circuit are also studied in order to demonstrate the slots and the CSRRs effect parameters on the behavior of the proposed filter. A prototype of the optimal filter is fabricated and measured.Theory and experiment results prove the validity of the new design procedure.
\end{abstract}

\section{Introduction}

Parallel-coupled line microstrip filters are known as one of the most commonly used designs in practical wireless communication and microwave applications, due to their design simplicity, planar structure, and relatively wide bandwidth [1]. However, this type of filter suffers from spurious responses that limit their performances and their integration in RF front-end of wireless microwave systems. Hence, these filters require high selectivity [2] and spurious suppression [3-4]. These spurious appear in the outof-band rejection at the even- and odd-mode characteristics, which produce, a poor out-of-band rejection. For this reason, various methods have been proposed to overcome the aforementioned challenges. To eliminate the spurious suppression many techniques are used. Some authors for example, proposed an open stubs with improvements in over coupled end sections and period perturbation to get multi-spurious suppression, [5]. Others integrated an open stub of the band pass filter $(B P F)$ over the antenna slot to suppressing the high-order spurious, [6]. On other hand, many works used the meandered quarter-wavelength resonators to suppress the unwanted harmonics [7]. To enhance the filter selectivity in out-of-band rejection, several techniques have been used to face this kind of problems. For example, [8] integrated two short-circuited stubs with one quarter-wavelength to develop quadruple-mode $M M R$ filter. Besides, [9] proposed a quarter wavelength shortcircuited stub to be added at each side of the central coupled line, with the aim to increase significantly the selectivity of both edges of the passband filter. Although, [10] adopted a defected ground structure $(D G S)$ technologies. However, [11] proposed transmission zeros $(T Z s)$ and [12] adjusted multi-stage cascade resonators. The selectivity enhancement, and the harmonic suppression were treated in [13] by seven transmission zeros $(T Z s)$. Although, most of these approaches still need some enhancements. In this paper, we propose a design and analysis of a performed wideband bandpass filter based on complementary split ring resonators $(C S R R s)$ and slot resonators. The proposed filter can be used for different technology, in particular for the satellite communication systems. The main objectives of this paper is to improve the filter selectivity, suppress the spurious response and eliminate the transmission at high frequency. To achieve this, firstly, the parallel-coupled line microstrip bandpass filter is optimized to cover the microwave C-band from 4.6to7.5 $\mathrm{GHz}$. Secondly, two symmetrical resonator types such as Slot and CSRR elements are analyzed, regarding the second harmonics reduction. After that, these parasitic elements are added to the circuit to yield spurious suppression and selectivity improvement for the desired resonance frequencies. These elements are loaded on the fiter design in two steps. exactly in the transmission line of $50-\Omega$ and below it respectively, see figures below.The experimental validation is done for the first case related to the spurious suppression and the selectivity enhancement. To widen the rejection between these two dips, two additional $C S R R s$ particles with close resonances are 
etched to eliminate the unwanted transmission at high frequencies. An L-C equivalent circuit model is also presented and calculated for all design procedures to demonstrate the performance of the proposed filter. The optimized design structure offers a miniaturized C-band bandpass filter with better out-of-band rejection.

\section{Bandpass filter design with spurious suppression}

\subsection{Geometry of the proposed filter}

Fig. 1. shows a general layout of proposed three-pole Chebyshev parallel coupled line microstrip band-pass filter. This structure implements the Roger RT/DuroidRO3010 substrate with the dielectric constant of 10.2 and thickness of 1.27. The parameter dimensions of the optimized filter are fixed at the operating frequency centred at $6 \mathrm{GHz}$ with the equal ripple of $0.1 d B$. These ripples were calculated by the tools developed in [10] and optimized by the CST MWs Electromagnetic Simulator. The corresponding evenand odd-mode impedance characteristics are $Z_{0 e}=94 \Omega$, $Z_{0 o}=40 \Omega$ for sections 1,4 and $Z_{0 e}=72.3 \Omega$, $Z_{0 o}=34.2 \Omega$ for sections 2,3 . The L-C elements for serial

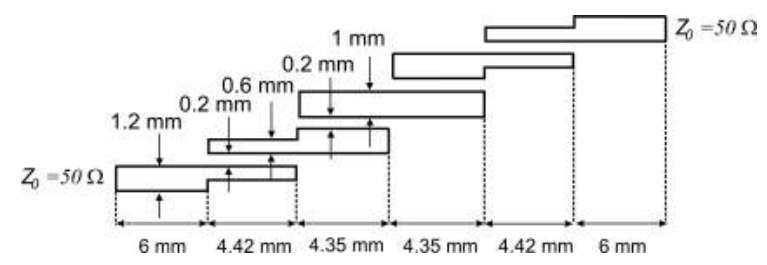

Figure 1: Configuration of the proposed C-band parallel coupled bandpass filter

and parallel coupled bandpass filter are calculated using the Chebyshev function, [1] as can be shown in the following equations:

$$
\begin{gathered}
L_{s}=\frac{F B W \cdot \omega_{0}}{Z_{0} \cdot g} \quad ; \quad C_{s}=\frac{F B W}{Z_{0} \cdot g \cdot \omega_{0}} \\
L_{p}=\frac{F B W \cdot Z_{0}}{\omega_{0} \cdot g} \quad ; \quad C_{p}=\frac{g}{F B W \cdot Z_{0} \cdot \omega_{0}}
\end{gathered}
$$

Where $g$ is the Chebyshev element and $F B W$ is the fractional bandwidth, $F B W=\frac{\omega_{1} \cdot \omega_{2}}{\omega_{0}}$ with $\omega_{0}=\left(\omega_{1} \cdot \omega_{2}\right)^{0.5}$

The calculated values of the L-C components for the circuit presented in Fig.3, are: $C_{1}=C_{3}=0.218 p F$, $C_{2}=1.460 \mathrm{pF}, L_{1}=L_{3}=3.283 \mathrm{nH}, L_{2}=0.489 \mathrm{nH}$.

The frequency response of the resulting bandpass filter is plotted in Fig. 2, indicating a good agreement between simulation and the equivalent circuit model developed in Fig.3. From Fig.2, it is observed also that the filter covers the microwave C-band from $4.6-7.5 \mathrm{GHz}$ with low passband insertion loss of $0.6 \mathrm{~dB}$. although, the results obtained are acceptable. from the figure it is clear to appreciate the presence of an unwanted harmonic step band of $11.1-13 \mathrm{GHz}$.

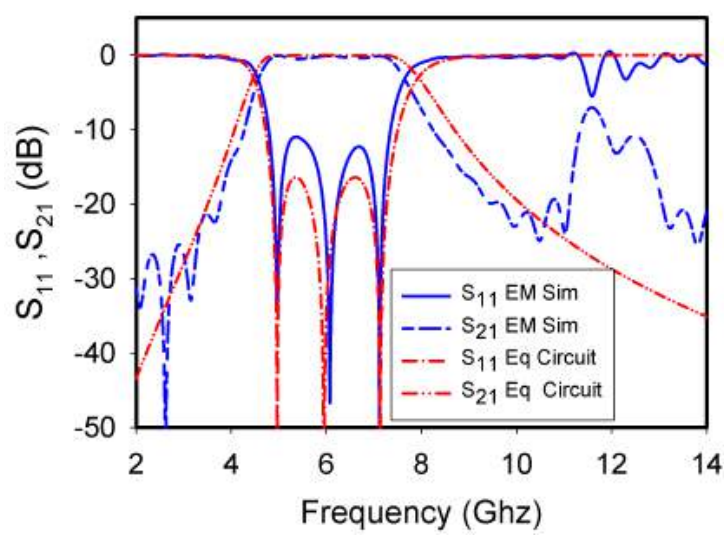

Figure 2: Electrical response of the conventionnel UWB parallel coupled bandpass filter

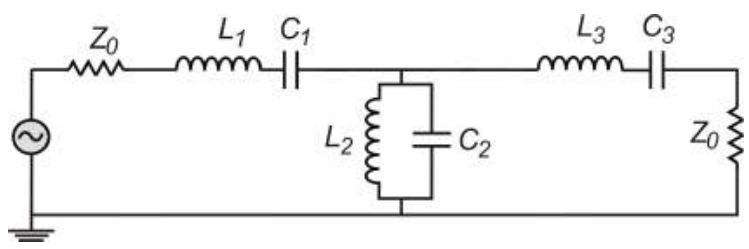

Figure 3: Equivalent circuit model of the conventionnel UWB parallel coupled bandpass filter

\subsection{Spurious Suppression using Slot/CSRR resonators}

In order to enhance the filter response presented in the previous section by eliminating the second harmonics response, two different techniques are introduced. The first technique, is presented in Fig.4 it consists of embedding open stubs in the transmission feed with the aim to obtain the rejection in the desired frequency band. The frequency rejected depends on the length of the stub [11]. As can be shown from Fig.4, the second technique its based on the insertion of CSRRs parasitic elements in the ground to provide negative effective permittivity and to produce a sharp rejection [12]. These techniques are introduced separately to be compared to each other. It can be noticed that, the rejection enhancement is achieved in both cases. The resonance frequency for the $C S R R s$ can be calculated by the fellow equation:

$$
\omega_{0}=\sqrt{ } \frac{2}{\pi r_{0} L_{0} C}
$$

Where $r_{0}$ is the average radius of the two annular slots, $L_{0}$ is the inductance per unit length between the annular slots and $C$ is the total capacitance of the $S R R$. 

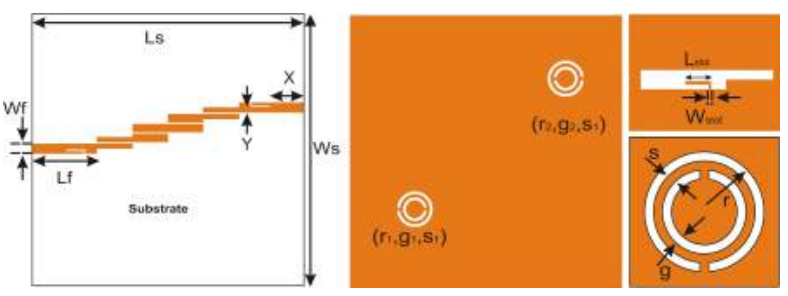

Figure 4: Configration of the filter with stubs and CSRRs $\left(r_{1}=r_{2}, g_{1}=g_{2}, s_{1}=s_{2}, x=4.2 \mathrm{~mm}, y=0.3 \mathrm{~mm}\right.$, $L_{\text {slot }}=2.4 \mathrm{~mm}, W_{\text {slot }}=0.2 \mathrm{~mm}$ )

To validate the proposed designs, several filter prototypes are fabricated using Rogers $R O-3010$ substrate. The photograph of the manufactured prototypes is presented in Fig. 5.
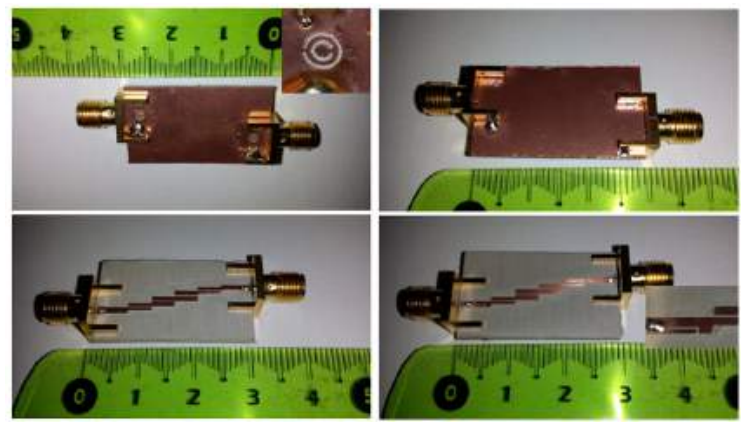

Figure 5: Photograph of the proposed filters : Top and butom layers with CSRRs (left), Top and butom layers with Slots(right)

The effect of the proposed resonators on the spurious suppression, compared to conventional filter design is presented in Fig. 6. It is clear that the open stub technique offers more than $20 d B$ rejection level in the undesired passband of $(11-13) \mathrm{GHz}$ with a passband insertion loss of $1 \mathrm{~dB}$, while the CSRR method produces rejection level more than $20 \mathrm{~dB}$ with low passband insertion loss of $0.8 d B$. Note that the differential between simulation and measurement results is due to the fabrication tolerances, as well as the location of the $C S R R$, in the vicinity of the soldered microstrip launcher ports.

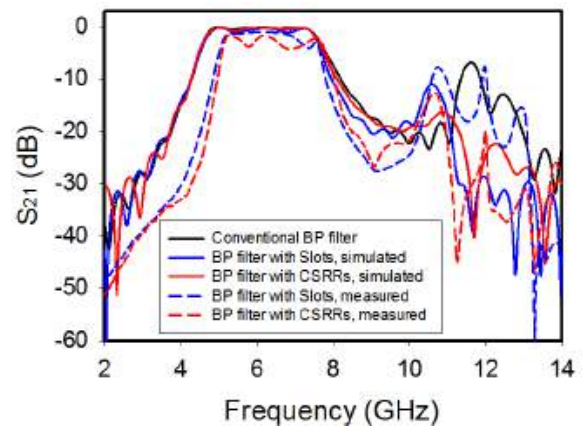

Figure 6: Simulated and measured insertion loss of the proposed C-band bandpass filter
To enhance the proposed filter presented previously, two-slots are etched on the feed line and $6-C S R R s$ with three close resonance frequencies are collocated exactly bellow the transmission line. It should be noted that the whole structure design remain the same in terms of symmetry and size.

\section{Bandpass filter with performed out-of-band rejection}

\subsection{Miniaturized Slot- and CSRR-loaded bandpass fil- ter with enhanced selectivity}

Based on the same filtering structure, we propose adjusting the $C S R R$-grounded element to operate at $8.75 \mathrm{GHz}$ in order to improve the filter selectivity at this frequency. This configuration offers a miniaturization capability in terms of selectivity enhancement and spurious suppression. Fig. 7 illustrates the equivalent circuit model of the parallelcoupled microstrip band pass filter $(P C M B P F)$ loaded with Slots and CSRRs resonators. $\omega_{i}=\sqrt{ } \frac{1}{L_{i}\left(C_{c i}+C_{i}\right)}$, $(\mathrm{i}=4,5,6,7, \ldots)$ is the angular resonance frequency of transmission line loaded with single $C S R R$, [14,15]. From [16] and [17]:

$$
\begin{gathered}
C_{c}=\frac{\varepsilon}{d} \\
C=4\left(\frac{\varepsilon_{0}}{\mu_{0}}\right) L s \\
L=\frac{L_{0}}{4}=\frac{\pi r_{0} L_{p u l}}{2}
\end{gathered}
$$

Where $C_{c}$ is the coupling capacitance between the line and the $C S R R / S l o t s, \mathrm{C}$ and $\mathrm{L}$ being the reactive elements, $d$ is the space between the rings, $r_{0}$ is the average radius and $L_{p u l}$ is the per unit length capacitance between the rings.

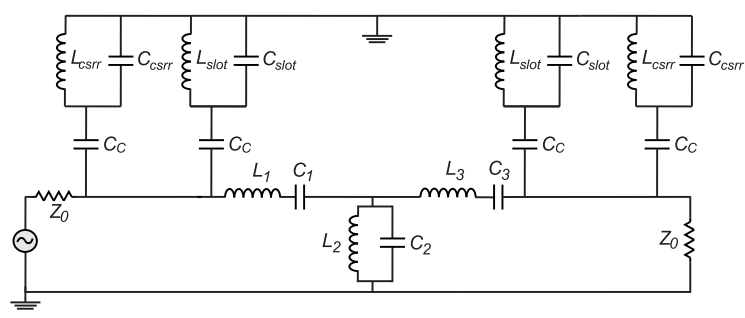

Figure 7: Equivalent circuit model with two $C S R R$ and slot-loaded in the ground and the transmission lines respectively

Fig.8. depicts the insertion loss of the model presented in Fig.4. As can be seen, good agreement between EM simulation and equivalent circuit results are achieved with high miniaturization capability in terms of selectivity and harmonics reduction for both resonance frequencies of 8.75 and $11.56 \mathrm{GHz}$ respectively. To widen the rejection between both $S_{21}$ dips and eliminate the undesirable transmission at high frequencies, four $C S R R$ particles should be added with close resonance frequencies. 


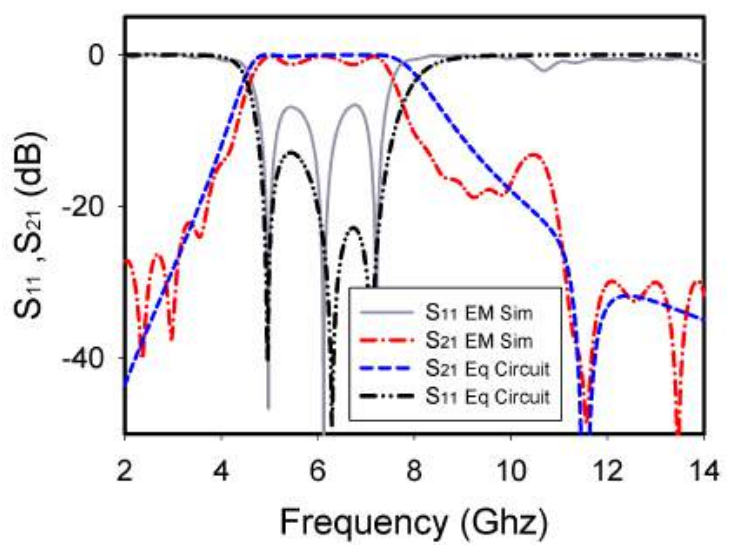

Figure 8: Insertion loss of the proposed C-band bandpass filters with Slot and $2-C S R R s$

Based on the CSRR resonance frequency expression given in [18], the calculated components values of the equivalent circuit model depicted in Fig. 7, are: $C_{c s r r}=$ $0.04 p F, C_{c}=0.03 p F, C_{\text {slot }}=0.15 p F, L_{\text {csrr }}=$ $4.9 n \mathrm{H}, L_{\text {slot }}=1.27 \mathrm{nH}$.

$$
\omega_{g, \text { Slot }}=\sqrt{ } \frac{1}{L_{s_{l}}+C_{s_{l}}}
$$

Where $\omega_{g}$ is the angular resonance frequency of the transmission line loaded with single Slot.

\subsection{Bandpass filter rejection performance}

The optimized bandpass filter and loaded by slot resonators and $C S R R$ parasitic elements is presented in Fig. 9. These resonators are integrated with the aim to eliminate the unwanted transmission at high frequencies, in particular at $8.75 \mathrm{GHz}, 9.34,10.17$ and $11.56 \mathrm{GHz}$.

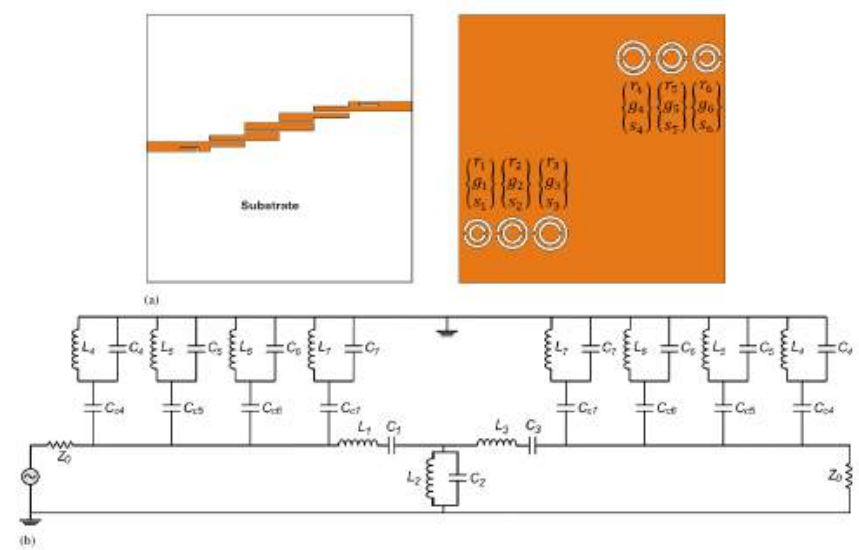

Figure 9: (a): Top and Bottom views of the optimized filter design, (b) : Correspondent equivalent circuit

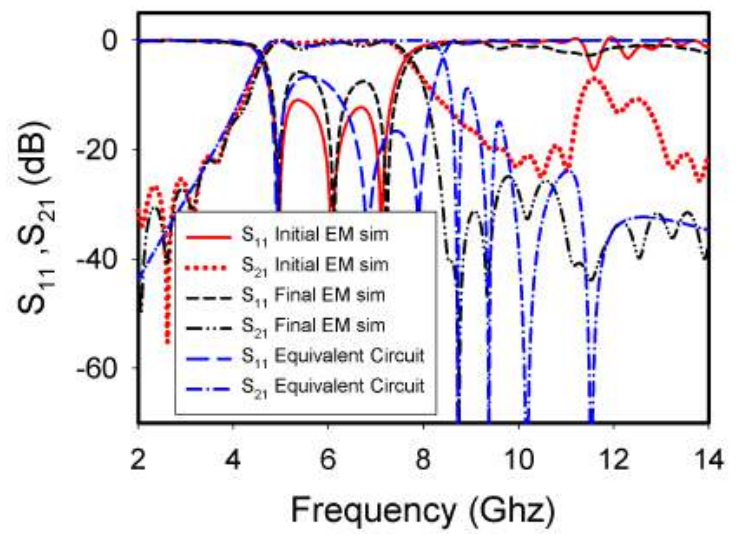

Figure 10: Frequency responses of the conventional and the proposed wideband bandpass filter for EM simulation and equivalent circuit model

The frequency response depicted in Fig.10 indicates that the filter covers the microwave C-band $(4.6-7.5 \mathrm{GHz})$ with a wide rejection performance within the whole frequency band from 8 to $14 \mathrm{GHz}$ with $S_{21} \prec 20 d B$, compared to the filter of Fig.1. A high selectivity at $8.75 \mathrm{GHz}$ is also obtained with harmonics suppression around $11.56 \mathrm{GHz}$. In addition frequency response of the calculated equivalent circuit matches very well with EM simulation, regarding both selected and rejected frequency bands.

The updated component values of equivalent circuit $\mathrm{L}$ and $\mathrm{C}$ for serial and parallel combination with three CSRRs are: $C_{6}=0.1 \quad p F, C_{c 6}=0.08 p F$, $C_{7}=0.035 p F, C_{c 7}=0.029 \quad p F, L_{6}=1.36 n H$, $L_{7}=4.5 \mathrm{nH}$.

Table 1. shows the parameter values of the three CSRR elements with their corresponding radiation frequencies.

Table 1: Parameter and frequency values of $3-C S R R s$

\begin{tabular}{ccccc}
\hline $\begin{array}{c}\text { Coupled } \\
\text { Rings }\end{array}$ & $\begin{array}{c}\text { Frequency } \\
(\mathrm{GHz})\end{array}$ & $\begin{array}{c}\text { Space } \\
\text { Gap(mm) }\end{array}$ & $\begin{array}{c}\text { Diameter } \\
\text { of Outer } \\
\text { Ring(mm) }\end{array}$ & $\begin{array}{c}\text { Diameter } \\
\text { of Inner } \\
\text { Ring(mm) }\end{array}$ \\
\hline 1 & 8.75 & 0.2 & 2.4 & 1.6 \\
2 & 9.34 & 0.2 & 2.2 & 1.4 \\
3 & 10.17 & 0.2 & 2 & 1.2 \\
\hline
\end{tabular}

While Table 2. summarized $L$ and $C$ parameter values of the equivalent circuit related to the filter with three $C S R R s$ and Slot. 
Table 2: Element parameters of the structures shown in Fig. 9

\begin{tabular}{ccccc}
\hline & 1-CSRR & 2-CSRR & 3-CSRR & Slot \\
\hline$F_{i}(\mathrm{GHz})$ & 8.75 & 9.34 & 10.17 & 11.56 \\
$L_{i}(\mathrm{nH})$ & 4.9 & 4.5 & 1.36 & 1.27 \\
$C_{i}(\mathrm{pF})$ & 0.04 & 0.035 & 0.1 & 0.15 \\
$C_{c} i(\mathrm{pF})$ & 0.03 & 0.029 & 0.08 & 0.03 \\
\hline
\end{tabular}

To show the slots and parasitic elements $C S R R$ effects, a study of the current distribution of proposed design in Fig. 9 is taken into consideration.

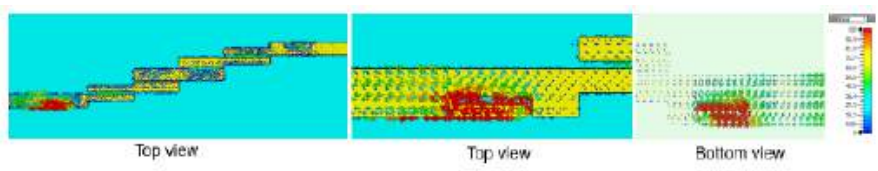

(a)

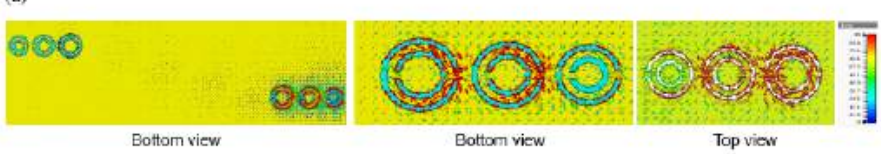

(b)

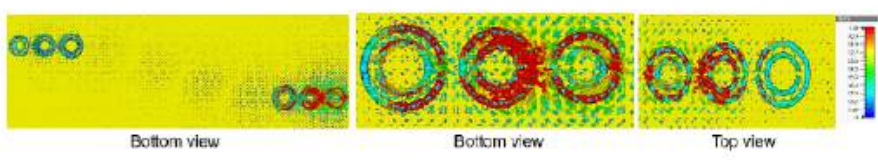

(c)

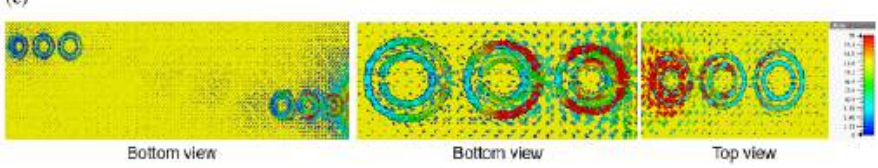

(d)

Figure 11: Current distribution of the proposed filters at : (a): $11.56 \mathrm{GHz}$, (b): $8.75 \mathrm{GHz}$, (c): $9.34 \mathrm{GHz}$ and (d): $10.17 \mathrm{GHz}$

The behavior of the current is presented in Fig. 11 for the operating frequencies of $(8.75,9.34,10.17$ and $11.56 \mathrm{GHz}$ ), of the rejected bands. It can be noticed that the quasi-static resonance frequencies of the slot/CSRR elements are located just in these four frequencies, which proves that the transmission is then eliminated for those close frequencies resulting in a wide rejection performance.

In Table 3 we compare the performance of the proposed band pass filter with other filters published in literature. We can observe that the developed filter in this paper is more suitable and presents more advantages than other filters cited in the literature. It's clear that our filter is characterized by a small size, wide rejection bandwidth and high selectivity level. We note also that the technique used in this paper permits to eliminate the transmission at high frequency and suppress the spurious responses.
Table 3: Comparison of the proposed filter and similars published in literature

\begin{tabular}{clllll}
\hline Ref. & Technique & $\begin{array}{c}\text { Size } \\
\left(\mathrm{mm}^{2}\right)\end{array}$ & $\begin{array}{c}\text { Wide } \\
\text { Stopband }\end{array}$ & $\begin{array}{c}\text { Frequency } \\
(\mathrm{GHz})\end{array}$ & $\begin{array}{c}\text { Rejection } \\
\text { Level(dB) }\end{array}$ \\
\hline$[19]$ & U-slot & 508.2 & Yes & 2.8 & 35 \\
& DGS & & & 10 & 28 \\
& & & & 3.5 & 28.6 \\
{$[20]$} & T-shaped & 320 & No & 7.5 & 25.1 \\
& L- DMS & & & & \\
& & & & 5.2 & 22,7 \\
{$[21]$} & Stubs & 419.9 & Yes & 10.85 & 25 \\
& & & & 12.42 & 56 \\
{$[22]$} & Stubs & - & No & 6.6 & 32 \\
& & & & & \\
{$[23]$} & E-shaped & 476 & Yes & 5.8 & 15 \\
& & & & & \\
& & & & 8,75 & More than \\
This & Slot & \multirow{2}{*}{291.04} & Yes & 9,34 & 70 for all \\
Work & CSRR & & & 10,17 & frequencies \\
& & & & 11,56 & \\
\hline
\end{tabular}

\section{CONCLUSION}

In this paper, a compact wideband parallel coupled line microstrip bandpass filter with improved out-of-band rejection performance is proposed for C-band satellite communications. By loading a slot and $C S R R$ parasitic elements with close resonances in both filter sides, we achieved a high miniaturization capability in term of out-of-band rejection without affecting the in-band filtering characteristics. This miniaturization concerns the spurious suppression, the selectivity improvement and the wide rejection performances. The simulation results, equivalent circuit modeling and experimental validation indicate that the proposed techniques are a good candidate to miniaturize parallel coupled line microstrip bandpass filters for the required applications. This design idea can be extended to load the CSRR resonators in the ground plane bellow the coupled lines by considering the odd and even characteristics modes.

\section{References}

[1] A. Naghar, O.Aghzout, A.V. Alejos, F. Falcone,Synthesis Design of BandPass Filter for UWB Applications with Improved Selectivity ,ACES JOURNAL, Vol. 31, No. 1, January 2016.

[2] G.Wua, X.Zhang, and W. Li, "Novel bandpass filter with high selectivity and very wide stopband using open stub loaded and DGS" IEICE Electronics Express, Vol.14, No.16, 1-6. 
[3] R. C. Hadarig , M. E. de C Gomez and F. Las-Heras, "A Compact Band-Pass Filter with High Selectivity and Second Harmonic Suppression" Materials, ISSN 1996-1944,2013.

[4] A. Naghar, O. Aghzout, A. V. Alejos, M. G. Sanchez and M. Essaaidi "Design of compact multiband bandpass filter with suppression of second harmonic spurious by coupling gap reduction" Journal of Electromagnetic Waves and Applications, Vol. 29, No. 14, 1813-1828, 2015.

[5] M. L. Roy and A. Pérennec, "Spurious Responses Suppression of Parallel Coupled-Lines Microstrip Bandpass Filters: Comparison and Improvements of Planar Approaches" HAL , Id: hal-00468122, 2010.

[6] L.Yang, P. Cheong, LiangHan, W. W. Choi, K.W. Tam, and KeWu,'Miniaturized Parallel Coupled-Line Filter-Antenna with Spurious Response Suppression" IEEE Antennas and Wireless Propagation Letters, Vol. 10, 2011.

[7] Y.L. Lu, S.Wang, T. Gu, P.Cao, and K. Li,'A Miniaturize Bandpass Filter with Harmonic Suppression Using Meandered Quarter-Wavelength Resonators" International Journal of Antennas and Propagation , ID 916927, 6 pages, Vol 2014.

[8] S. W. Wong, and L. Zhu,"Quadruple-Mode UWB Bandpass Filter With Improved Out-of-Band Rejection" IEEE Microwave and Wireless Components Letters ,Vol. 19, No. 3, March 2009.

[9] H. N. Shamanp, "Design of a Compact C-band Microstrip Bandpass Filter for Satellite Communications Applications" IEEE ,978-1-4673-1989-8/12 , 2012.

[10] W. Jiang, L. Zhou, A.M. Gao, W. Shen, W.Y. Yin and J.F. Mao, "Compact dual-mode dual-band balun filter using double-sided parallel-strip line" Electronics Letters, Vol. 48 No. 21, 11th October 2012.

[11] K. Wang, S.W. Wong, G.H.Sun, Z. N. Chen, L. Zhu, and Q. X. Chu, "Synthesis Method for SubstrateIntegrated Waveguide Bandpass Filter With EvenOrder Chebyshev Response" IEEE Transactions on Components, Packaging and Manufacturing Technology, Vol. 6, no. 1, January 2016.

[12] W. Feng, W. Che, S. Shi, and Q. Xue, "High Selectivity Wideband Bandpass Filter Based on Transversal Signal-Interaction Concepts and T-Shaped Structure" IEEE Microwave and Wireless Components Letters , Vol. 22, No. 11, November 2012.

[13] T. Huang and Z. Shao, "A size-miniaturized bandpass filter with selectivity-enhanced and high harmonic suppression performance" International Journal of Microwave and Wireless Technologies, page 1 of 7, 2017.
[14] Lei Zhu," Guided-wave characteristics of periodic coplanar waveguides with inductive loading-unitlength transmission parameters ", IEEE Trans. Microwave Theory and Tech., vol. 51, no. 10, pp. 21332138, Oct. 2003.

[15] L. Su, J. Bonache, J. Mata-Contreras and F. Martín, "Modeling and Applications of Metamaterial Transmission Lines Loaded With Pairs of Coupled Complementary Split-Ring Resonators (CSRRs)", IEEE Trans. Antennas and Wireless Prop. Lett. , vol. 15, pp. 154-157, 2016.

[16] J. Zhang and X.-W. Sun, "Harmonic Suppression of Branch-Line and Rat-Race Coupler using Complementary Spilt Ring Resonators (CSRR) Cell”,Progress In Electromagnetics Research Letters, Vol. 2, 73-79, 2008.

[17] Juan Domingo Baena, Jordi Bonache, Ferran Martín, Ricardo Marqués Sillero, Francisco Falcone, Txema Lopetegi, Miguel A. G. Laso, Joan García-García, Ignacio Gil, Maria Flores Portillo, and Mario Sorolla, "Equivalent-Circuit Models for Split-Ring Resonators and Complementary Split-Ring Resonators Coupled to Planar Transmission Lines", IEEE Transactions on Microwave Theory and Techniques, Vol. 53, No. 4, April 2005.

[18] D. Kajfez. and. S. Govind, " Effect of Difference in Odd- and even- mode Wavlengths on a ParallelCoupled Bandpass Filter ", IEE Electronics Letters, vol. 11, no. 5, pp. 117-118, Mar. 1975.

[19] D. A. Salem, Ashraf. S. Mohra, A. Sebak , " A compact ultra wideband bandpass filter using arrow coupled lines with defected ground structure ", Journal of Electrical Systems and Information Technology, Volume 1, Issue 1, Pages 36-44, 2014.

[20] Zheng, X.; Pan, Y.; Jiang, T, ”UWB Bandpass Filter with Dual Notched Bands Using T-Shaped Resonator and L-Shaped Defected Microstrip Structure ", Micromachines , 9, 280, 2018.

[21] Weng, M.-H.; Hsu, C.-W.; Lan, S.-W.; Yang, R.-Y., "An Ultra-Wideband Bandpass Filter with a Notch Band and Wide Upper Bandstop Performances ", Electronics, 8, 1316, 2019.

[22] H. Shaman and J. Hong, " Asymmetric ParallelCoupled Lines for Notch Implementation in UWB Filters ", IEEE Microwave and Wireless Components Letters, vol. 17, no. 7, pp. 516-518, July 2007.

[23] H. Peng, Y. Luo, and J. Zhao , " Compact Microstrip UWB Bandpass Filter with Two Band-Notches for UWB Applications", Progress In Electromagnetics Research Letters, Vol. 45, 25-30, 2014. 